Southern Illinois University Edwardsville

SPARK

SIUE Faculty Research, Scholarship, and Creative Activity

Winter 1-30-2014

\title{
A Non-equilibrium Approach to Temperature Calibration for Tungsten Filament Atomic Spectrometry
}

\author{
Edward C. Navarre \\ Southern Illinois University Edwardsville, enavarr@siue.edu \\ Paige Wallace \\ Southern Illinois University Edwardsville
}

Follow this and additional works at: https://spark.siue.edu/siue_fac

Part of the Analytical Chemistry Commons

\section{Recommended Citation}

Navarre, Edward C. and Wallace, Paige, "A Non-equilibrium Approach to Temperature Calibration for Tungsten Filament Atomic Spectrometry" (2014). SIUE Faculty Research, Scholarship, and Creative Activity. 142.

https://spark.siue.edu/siue_fac/142

This Article is brought to you for free and open access by SPARK. It has been accepted for inclusion in SIUE Faculty Research, Scholarship, and Creative Activity by an authorized administrator of SPARK. For more information, please contact magrase@siue.edu,tdvorak@siue.edu. 
Title: A Non-equilibrium Approach to Temperature Calibration for Tungsten Filament Atomic Spectrometry

Short title: Voltamperometric Temperature Study for Filament ETAAS

Abstract

A non-equilibrium approach voltamperometric temperature measurement for tungsten filament atomizers was developed to facilitate the transfer of thermal conditions between instruments that possess different power supply regulation modes and filaments. Large differences in the equilibrium and instantaneous temperatures were found during the pyrolysis and atomization steps of cadmium AAS analysis. By using the instantaneous temperature, the pyrolysis and atomization behaviors of cadmium were shown to be equivalent regardless of the power supply regulation mode. The pyrolysis conditions optimized for a $15 \mathrm{~V}$ filament were readily and accurately transferred to a $12 \mathrm{~V}$ filament by applying the non-equilibrium voltamperometric temperature model.

Introduction

Tungsten filaments have been investigated as an alternative atomization source for atomic absorption, emission, fluorescence, and as a vaporizer for sample introduction to ICP-OES and ICP-MS (1-5). The high heating rate of tungsten filaments afforded by their small mass and low specific heat is one of their desirable features for electrothermal atomic absorption and emission spectrometry. The small mass also makes it difficult or impossible to use a thermocouple to measure the temperature (6). 
As a result, temperature calibration of tungsten filament electrothermal atomizers has been a subject of study for many years. Various approaches exist in the literature including optical pyrometry, (7-10) blackbody emission by a two-line method, $(11,12)$ and voltamperometric methods $(6,8)$. At temperatures below the functional range of optical methods (less than $800 \mathrm{~K}$ ) the melting points of inorganic salts have been used to estimate the temperature. $(6,8,9)$.

Several temperature models have been developed; however, there is some disagreement in the correlation between temperature and electrical properties. For example, the temperature of the same model of a $15-\mathrm{V}$ lamp filament at $10 \mathrm{~A}$ has been reported as 2600 and $3415 \mathrm{~K}(10,13)$ and at $12 \mathrm{~V}$ has been reported as 2070 and 2960 $\mathrm{K}(8,14)$. Differences of about $600 \mathrm{~K}$ have been reported in the filament surface temperature between voltamperometric and optical pyrometric temperature models (8).

In the tungsten filament AAS literature analysis conditions are often presented in terms of electrical properties such as voltage, current, or power, rather than temperature. As a result, it is often challenging to implement a published method on a different instrument. More so, the use of electrical properties to define a method can present barriers to transferring conditions developed on one instrument and filament to another even within the same laboratory.

Most of the temperature studies on tungsten filament AAS deal with the equilibrium temperature of the filament. It has been argued that the filament heating rate and rate of analyte vaporization is more indicative of the atomic absorption signal than the equilibrium temperature of the filament (15). As an open atomizer, the tungsten filament also possesses a large spatial temperature gradient that strongly 
influences analyte signal intensity and the effects of concomitants. The temporal and spatial heterogeneity of the filament atomizer have a profound influence on the gasphase chemistry and analytical properties of the instrument.

It has been noted in the authors' laboratory that atomic absorbance signals often appear prior to the filament achieving its equilibrium temperature. This observation suggests that a non-equilibrium approach to temperature may be informative to develop strategies to operate tungsten filament atomizers in terms of temperature so that the thermal conditions are independent of the particular power supply or filament used. Voltamperometric temperature calibration is well suited to the task of monitoring the filament temperature to reveal the dynamic heating process.

The development of a temperature model adaptable to diverse tungsten filament instruments would simplify the transfer of methods between instruments and facilitate the investigation of new hardware. This work revisits voltamperometric temperature calibration and develops a simple method to estimate the filament temperature throughout the thermal program. The method will examine the applicability to different filament types and the two most common power supply regulation modes: current and voltage. Temperature profiles will illustrate the non-equilibrium nature of the tungsten filament during its temperature program. The differences in the apparent optimum thermal conditions under different power supply regulation modes provide insight into the non-equilibrium conditions present during pyrolysis and atomization. The successful transfer of analysis conditions for cadmium between two filaments with very different properties will be demonstrated. 


\section{Experimental}

Sample solutions were diluted from $1000 \mathrm{mg} \mathrm{L}^{-1}$ aqueous cadmium standards (PLCD-2X, Spex CertiPrep). All solutions were diluted to contain $0.2 \%(\mathrm{v} / \mathrm{v})$ nitric acid (trace metal grade, Fisher Scientific). A $0.2 \%(\mathrm{w} / \mathrm{v})$ ammonium phosphate matrix modifer was used in some samples. The stock solution was prepared from ammonium dihydrogen phosphate (99.999\%, Acros Organics) and diluted as needed in the samples. Samples were applied using a variable volume micropipettor (Eppendorf) to the $15-\mathrm{V}$ filament as $10 \mu \mathrm{L}$ aliquots and $4 \mu \mathrm{L}$ on the $12-\mathrm{V}$ filament. The dimensions of the $12-\mathrm{V}$ filament are smaller than the $15-\mathrm{V}$, and could only accommodate $4 \mu \mathrm{L}$ of liquid in the coiled part of the filament. The filament masses were determined by cutting filaments from their bases and weighing them individually.

The tungsten filament AAS instrument used in this study is very similar to that used for lead AAS studies (16). Tungsten filaments were taken from commercial $15 \mathrm{~V} /$ $150 \mathrm{~W}$ and $12 \mathrm{~V} / 50 \mathrm{~W}$ lamps (64633 HLX and 64610 HLX, Osram Sylvania). The glass bulb was removed to expose the bare filament. The filament was held in a commercial lamp socket (model TP56, Osram Sylvania) inserted in a cylindrical aluminum base. The base had feed-throughs for electrical connections and support gas, and allowed mounting to a linear translation stage (NT38-180, Edmund Optics) for height adjustment. The filament was enclosed by a custom T-shaped glass shroud (Chemglass). A schematic of the filament and enclosure is shown in Fig. 1. The glass enclosure possessed a dosing hole at the top and fused silica windows (NT48-201, Edmund Optics) at opposite ends (horizontally) for the transmission of the hollow cathode lamp beam. The base of the shroud was sealed around the aluminum mount. 
A support gas of $10 \%$ hydrogen in argon (Airgas) was introduced to the glass enclosure at $0.5 \mathrm{~L} \mathrm{~min}-1$. Two support gas inlets were located on opposite sides of the lamp socket.

Illumination for cadmium AAS was provided by a Cd hollow cathode lamp (model 45462, Jarrell-Ash) with a lamp current of $6 \mathrm{~mA}$ supplied by an HCL power supply (model 6800 , Oriel). Light from the HCL was collected and focused by a $50-\mathrm{mm}$ focal length fused silica lens (NT48-294, Edmund Optics). The filament was positioned at the focal point of the hollow cathode lamp beam such that the top of the filament was at the bottom of the spectrograph observation volume. A second equivalent lens refocused the light onto the entrance slit of a compact spectrograph (Maya 2000 Pro, Ocean Optics). The spectrograph effective bandpass in the wavelength range of the cadmium lines was $0.2 \mathrm{~nm}$. Data were recorded with an integration time of $25 \mathrm{~ms}$ at the wavelengths $228.8,226.5$, and $227.4 \mathrm{~nm}$ for the cadmium analytical line, background correction, and baseline correction, respectively.

The AAS signals were recorded with SpectraSuite (Ocean Optics) in strip chart mode. Background correction and signal integration was performed with GRAMS/AI (v.9, ThermoGalactic). Any signal intensity from broadband lamp emission was removed from the cadmium analytical and background signals by subtracting the 227.4 $\mathrm{nm}$ signal. The two-line background correction method was used to determine the net absorbance signal at $228.8 \mathrm{~nm}$. (17) The reported integrated area and peak height are the mean of 5 replicate measurements of each sample.

The tungsten filament was heated by a programmable power supply (model 1696, BK Precision). The power supply was programmed via serial port with a custom 
interface written in Visual Basic (Microsoft Visual Studio v. 9). Current from 0.1 to 10.0 amperes and voltage from 1.0 to 15.0 volts could be programmed for a desired length of time in seconds. The power supply was operated in both current regulated and voltage regulated modes. A representative thermal program for a $15 \mathrm{~V}$ filament is shown in Table 1 with current and voltage values that yield approximately equal filament temperatures.

The experiments with $15-\mathrm{V}$ filaments conducted to create the pyrolysis curves used a constant atomization setting of $4.5 \mathrm{~V}$ and $6.0 \mathrm{~A}$, for the respective regulation modes. Atomization curves were determined with a constant pyrolysis settings of $1.0 \mathrm{~V}$ and 3.5 A for unmodified samples, and 1.5 $\mathrm{V}$ and 4.0 A for phosphate modified samples. In all experiments the cleaning step was set at least $0.5 \mathrm{~V}$ or $0.5 \mathrm{~A}$ above the value for atomization to ensure complete removal of the sample aliquot.

Current and voltage measurements of the filament were recorded with a differential oscilloscope (model 3425, PicoScope) operated at $100 \mathrm{~Hz}$. The current was measured with a magneto-resistive sensor (NT-15, F.W. Bell) inserted in the circuit. Filament voltage was measured directly by the oscilloscope with leads placed as close to the filament as possible.

Correct placement of the voltage measurement points was important for achieving accurate measurements. The difference in voltage between the terminals of the power supply and the base of the filament was typically 0.2 to $1.0 \mathrm{~V}$, depending on the magnitude of the current. This voltage corresponds to 0.1 to $0.2 \Omega$ in the lead wires of the instrument compared to 0.1 to $1.3 \Omega$ in the filament. 
Pragmatic voltage measurements during analysis must be made at the base pins of the filament assembly. The resistance contribution of the pins and any additional length of wire is readily determined by simultaneous voltage measurements at the two circuit positions.

The low-current characterization of the two kinds of filaments was performed with an Agilent E3631A power supply. The current and voltage of the filament was measured with a high precision multimeter (model 189, Fluke). The power supply was used for the low-current measurements because of its superior accuracy at $0.1 \mathrm{~A}$ compared to the BK Precision power supply.

Method

The voltamperometric temperature model is based on the temperature dependence of the filament resistance. Assuming that the filament is a pure tungsten wire of length, $L$, and cross-sectional area, $A$, the resistance, $R$, can be related to the electrical resistivity, $\rho$, as

$$
R(T)=\rho(T) \frac{L}{A}
$$

Using Ohm's law, the resistance is easily measured as $R=v / i$. The temperature dependent electrical resistivity values of tungsten are readily available (18). Therefore, only the length and cross-sectional area of the filament need to be determined to relate resistance to resistivity and thus determine temperature. The ratio $L / A$ was determined using a low-current $(0.1 \mathrm{~A})$ to measure the resistance of multiple filaments at a known temperature. Forced air cooling was used to ensure that the filament temperature did not appreciably change. The measured filament resistance at room temperature and 
the electrical resistivity at the same temperature allowed the value of $L / A$ to be found by eq. 1.

Results

Electrical measurements at low current found an average resistance of $64.9 \pm 1$ $m \Omega(n=16)$ for the $15-V$ filament at $297.6 \mathrm{~K}$. This result yields a length to area ratio $(L / A)$ of $1.204 \times 10^{6} \pm 2.6 \times 10^{4} \mathrm{~m}^{-1}$. The result is $7.4 \pm 2.3 \%$ larger than that determined for the same type of filament by a different method (8). Although the filaments used in this study and in the previously published work are the same manufacturer part number, they were purchased more than 10 years apart. The mass of the $15 \mathrm{~V}$ filaments in this study were $0.093 \mathrm{~g}$, compared to $0.108 \mathrm{~g}$ reported previously. Since the mass was used to determine the literature value of $L / A$, the smaller measured mass would account for more than half of the difference in $L / A$. Experimentally, some of the variation in $L / A$ can be accounted for by the difficulty of placing the voltage probes accurately at the base of the filament; a shorter length of filament would yield a smaller resistance and consequently a larger value of $L / A$. In a worst-case analysis assuming all error to be positive, the difference in $L / A$ results in a maximum overestimate of $6 \%$ in the filament temperature versus literature. Physical expansion of the filament is not considered to be a major source of error in the $L / A$ value. The coefficient of thermal expansion in tungsten is $4.5 \mu \mathrm{m} / \mathrm{m} \mathrm{K}(19)$. Over a $3000 \mathrm{~K}$ change in temperature the thermal expansion results in a $1.4 \%$ change in length. The change in $L / A$ is then smaller than the standard deviation of the $L / A$ determination. Nevertheless, the two values of $L / A$ agree well for the purpose of estimating of the filament temperature. 
To develop a relationship between temperature and resistance, the current and voltage of several $15-\mathrm{V}$ filaments were measured under equilibrium conditions. Measurements were made with the power supply in both current- and voltage-regulated modes. The equilibrium resistance, $R(T)$, was calculated for each point. The equilibrium temperature was determined as the temperature corresponding to the $\rho(T)$ that satisfied eq. 1 yielding a resistance equivalent to the measured value. The temperature and resistance data are shown in Fig. 2 along with their fit to equation 2.

$$
T=23.59+2716 \times R^{0.8367}
$$

Non-linear least squares optimization was used to determine the coefficients in eq. 2. An exponential fit was found to have smaller residuals than polynomial fits up to $4^{\text {th }}$ order.

The voltamperometric calibration developed in this work can be used to express equilibrium temperature as a function of current, voltage, or power for comparison to other temperature models. The model is shown in Figure 3 as a function of applied voltage and current together with similar models from the literature. The temperaturecurrent model in Fig $3 \mathrm{~A}$ from the literature (12) is based on a two-wavelength blackbody emission measurement. A temperature-voltage model compiled from optical pyrometry data previously published $(8,9)$ is shown in Fig $3 \mathrm{~B}$. In both plots the standard deviation in the temperature estimate due to uncertainty in $L / A$ and the electrical measurements is $\pm 2 \%$. There is excellent agreement between data sets at temperatures up to $2500 \mathrm{~K}$. The voltampermetric model agrees well with the temperature-current equation within its proposed range. Above $2500 \mathrm{~K}$ the literature data shows that higher voltages were necessary to achieve the temperature maximum (about $3340 \mathrm{~K}$ ) than were found in this 
study. However, it was noted in the published data that calibration of the power supply was critical for achieving reliable temperature data and differences up to $20 \%$ in applied voltage were observed between supplies for the same temperature. Given the overall error profile of the two methods, they can be viewed as in good agreement.

The higher calculated temperatures above $2500 \mathrm{~K}$ may also be explained by the fundamental difference in voltamperometric versus optical pyrometric methods. While optical pyrometry measures the filament surface temperature, voltamperometry measures the temperature as an aggregate of the whole filament. The voltamperometric model neglects the effects of radiative heat transfer from the filament, which tends to cool the surface relative to the center. Thus, there is a tendency to overestimate the temperature under conditions at which the filament emissivity is large. Despite neglecting the effects of radiative cooling at high temperature, the voltamperometric model is computationally simple and successful at estimating the filament temperature with good accuracy compared to other methods.

The voltamperometric model allows the measured resistance of the filament to be converted into a temperature at any time in the thermal program. To this end, voltage and current oscilloscope traces were recorded for each experiment and converted to a temperature trace using eq. 2. From the view point of equilibrium temperature, each current or voltage setting on the power supply corresponds to a single temperature, as shown in Fig. 3. However, the relatively slow heating rate of the tungsten filament results in non-equilibrium conditions for much of the pyrolysis and atomization phase of the thermal program. Slow temperature increase during pyrolysis can be due to the inherent thermal properties of the filament as well as traces of solvent 
remaining on the filament, or the sample matrix pyrolysis. A similar effect was observed at the end of the drying phase. Completion of the solvent evaporation process was frequently accompanied by an approximately $60 \mathrm{~K}$ temperature increase.

Shown in Fig 4 are the temperature traces recorded during the 5-second pyrolysis step of a typical analysis for cadmium. Traces for current-regulated and voltage-regulated steps are shown. During the 5 second pyrolysis, the temperature steadily rises in both cases and never reaches an equilibrium value. According to the voltamperometric model, the nominal power supply settings of $1.0 \mathrm{~V}$ and $3.5 \mathrm{~A}$ would indicate an equilibrium temperature of $1130 \mathrm{~K}$ and $1350 \mathrm{~K}$, respectively. As shown in Fig 4, in both cases the filament achieves a maximum temperature of about $800 \mathrm{~K}$ at the end of the pyrolysis step. The large difference in the equilibrium and instantaneous temperature of the filament indicates the importance of considering the non-equilibrium themal behavior.

Pyrolysis and atomization curves for aqueous Cd samples were recorded with current regulation and voltage regulation to test the utility of the non-equilibrium approach to temperature. In Fig. 5, pyrolysis and atomization curves are plotted together in terms of both equilibrium and non-equilibrium state temperature calculated with the voltamperometric model. The non-equilibrium values correspond to the maximum temperature achieved during the respective steps of the thermal program. In Fig. $5 \mathrm{~A}$ the current-regulated curves reach their maximum values at much higher temperatures than the voltage-regulated curves and give the impression that the thermal conditions vary with power supply regulation mode. When the temperature scale is plotted with the non-equilibrium, measured temperatures in each step, the 
curves in Fig 5B result. In the non-equilibrium plot there is good agreement in the best conditions for pyrolysis and atomization between power supply regulation modes. As expected, the thermal conditions must be the same because the chemical species present on the filament in each experiment are the same.

In Fig 5B, the maximum pyrolysis temperature determined in both voltage and current modes for unmodified Cd samples was $800 \mathrm{~K}$, corresponding to $1.0 \mathrm{~V}$ and $3.5 \mathrm{~A}$. A similar experiment with ammonium phosphate as a modifier exhibited a maximum pyrolysis temperature of about $1050 \mathrm{~K}$ for both power supply modes (nominally $1.3 \mathrm{~V}$ and 4.2 A). The phosphate modified and unmodified results match well with values determined by GFAAS and tungsten filament AAS $(6,7,20)$. In both power supply modes, the minimum atomization temperature was $1400 \mathrm{~K}$ and $1750 \mathrm{~K}$ for unmodified and modified Cd samples, respectively. In electrical terms, these temperatures correspond to2.5 $\mathrm{V}$ and 5.6 $\mathrm{A}$ (unmodified) and 3.5 $\mathrm{V}$ and 6.0 $\mathrm{A}$ (phosphate modified). The minimum atomization temperatures are about 200 to $300 \mathrm{~K}$ lower than the atomization temperature typical of GFAAS; however, lower atomization temperatures have been observed previously with $\mathrm{Cd}$ analysis by tungsten filament AAS and attributed to the open configuration of the filament. (10)

The ability of the temperature model to predict experimental conditions on a different filament was tested by performing cadmium analysis with a $12-\mathrm{V}$ filament. The lower voltage filament is significantly smaller and lighter $(0.018 \mathrm{~g})$ than the $15-\mathrm{V}$ filament $(0.093 \mathrm{~g})$. It has a current maximum of about $4.6 \mathrm{~A}$ compared to $10.8 \mathrm{~A}$ for the $15-\mathrm{V}$ filament. The $12-\mathrm{V}$ filament was initially characterized by recording the temperature profile of current steps at $0.1 \mathrm{~A}$ intervals over the range 0.1 to $4.8 \mathrm{~A}$. From the room 
temperature resistance of the filament, the length to cross sectional area ratio $(L / A)$ was determined to be $2.48 \times 10^{6} \pm 6.0 \times 10^{4} \mathrm{~m}^{-1}(\mathrm{n}=6)$. Using non-linear least squares analysis, the temperature-to-resistance relationship was found to be $T=27.2+1480 \times$ $R^{0.838}$.

In each temperature profile of the $12-\mathrm{V}$ filament, the temperature after 3 and 5 seconds was tabulated as an estimate of the maximum atomization and pyrolysis temperatures, respectively. From these estimates, current settings of 1.4, 1.5, 1.6, 1.7, and $1.8 \mathrm{~A}$ were selected prior to the experiment to mimic the data collected on the $15-\mathrm{V}$ filament. It was predicted that the maximum pyrolysis temperature for the $12-\mathrm{V}$ filament would occur at the 1.5 A setting. Shown in Fig. 6 are the pyrolysis curves for the 12-V filament under current regulation and the $15-\mathrm{V}$ filament under voltage regulation. The results show a maximum pyrolysis temperature of $1090 \mathrm{~K}$, corresponding to $1.6 \mathrm{~A}$. The temperature difference between the predicted and experimental values of current is only $40 \mathrm{~K}$ and is within the experimental error of the temperature estimates.

The minimum atomization temperature was similarly estimated and a current of 2.2 A was predicted. The atomization curves in Fig 6. show good agreement between the two filaments. The minimum atomization temperatures are estimated as $1750 \mathrm{~K}$ in both cases. At higher atomization temperatures both filaments exhibit a small decrease in the integrated area and improved precision at the highest temperatures.

In Fig. 5, the current-regulated and voltage-regulated heating programs result in the same minimum atomization temperature and approximately the same integrated area. However, the temperature profiles during atomization are quite different. The temperature profile and corresponding net absorbance profiles are shown in Fig. 7 for 
equivalent thermal conditions under current and voltage regulation. The voltageregulated program increases the temperature more rapidly and produces a well-defined absorbance feature about 1.1 seconds after the start of the atomization step. In the current-regulated example, the temperature increase is slower and the absorbance maximum occurs at about $2.4 \mathrm{~s}$ after the start of atomization. Although the two atomization steps result in equivalent integrated area and reach their maxima at the same temperature, the rate of heating has a strong influence on the peak height and width. The slower heating in current-regulated mode occurs because the filament temperature increases, principally, by Joule heating. At constant current, the process is controlled by the temperature coefficient of resistance. Heating in voltage-regulated mode is relatively faster because the small initial filament resistance, results in a large initial current. The applied current gradually decreases as the resistance increases to maintain constant voltage.

The differences in regulation mode are most evident when peak height is used for quantitation instead of area. In Fig 8, the atomization curves of the 15- and 12-V filaments are plotted as peak height. Under voltage regulation, the peak height reaches a maximum at $2400 \mathrm{~K}$. For both types of filament, the curve for current regulated atomization does not reach a maximum at temperatures over $2550 \mathrm{~K}$. Clearly, the effect is due to the dual proportionality of heating rate and number of analyte atoms on peak height (21).

\section{Conclusions}

A non-equilibrium approach to voltamperometric temperature measurement on a tungsten filament atomizer has been developed to facilitate the transfer and comparison 
of thermal conditions between different power supply regulation modes and filaments. The voltamperometric calibration model matches well with literature models based on other methods. The proposed method of characterizing the $L / A$ ratio of filaments readily accounts for variations in the production properties of the filament (e.g., total mass or diameter). For both of the filament types used in this study, the precision of the $L / A$ measurement was $2.3 \%$ and indicated good reproducibility in the batch of filaments. However, comparison of the filament mass of the same model of $15-\mathrm{V}$ filament purchased more than 10 years apart reveals a $14 \%$ decrease in mass. Thus, the long term reproducibility of $L / A$ for a particular commercial filament should be considered with caution.

The instrumentation needed for voltamperometric measurements is simple to implement on a tungsten filament atomizer. The initial characterization of a new filament or power supply is rapid. Only a series of voltage and current measurements are necessary to build a temperature model that permits transfer of a thermal method. The characterization of new types of filaments is also simple and requires only a stable low-current source and voltage measurement.

By observing the temporal profile of temperature throughout all steps of the thermal program, it is evident that analyte pyrolysis and atomization are often nonequilibrium processes. The equilibrium state temperature leads to apparent large differences in the best thermal conditions between power supply regulation modes. Plotting the filament temperature in terms of the measured (i.e., non-equilibrium) temperature yields consistent pyrolysis and atomization curves regardless of the power supply control mode. The non-equilibrium state temperatures for the pyrolysis and 
atomization of cadmium agree with published values for phosphate modified and unmodified samples.

The pyrolysis and atomization conditions developed for a 15-V filament in voltage regulated mode were readily and accurately transferred to a $12-\mathrm{V}$ filament in current regulated mode with a modest amount of filament characterization. These are very different instrumental conditions with respect to power supply and filament properties. It was possible to avoid a complete characterization of the new instrument by using the non-equilibrium state temperature to predict the pyrolysis and atomization conditions.

The proposed method for transferring thermal conditions between different instruments is most effective when integrated absorbance is used as the quantitative figure. When using peak height, the influence of the power supply's heating rate could not be completely removed by the non-equilibrium approach to temperature. Nevertheless, the non-equilibrium state temperature offers a more accurate representation of the thermal conditions for peak height data.

The application of the proposed method for predicting thermal conditions on current regulated power supplies requires some caution. Due to gradual mass loss and localized migration of tungsten at high temperature, the filament resistance will gradually increase with use. As a result, a given current will yield a higher temperature as the filament ages. The voltamperometeric method will be able to sense the increase in temperature over the filament lifetime and prompt the operator to adjust the current appropriately.

Relative to other temperature calibration approaches in the literature, the method presented in this work has the advantage of operating over the entire temperature range 
of the filament. It is able to sense the temperature of a filament loaded with aqueous solvent and the temperature increase that occurs as solvent evaporation finishes. In this way, the end of the drying step can be observed. Generally, the non-equilibrium approach to temperature described in this work provides a convenient way to report thermal conditions for tungsten filament atomizers that permits the transfer of methods to other instrumental hardware.

\section{References}

1 Bruhn, C. G.; Bustos, C. J.; Saez, K. L.; Neira, J. Y. and Alvarez, S. E. A comparative study of chemical modifiers in the determination of total arsenic in marine food by tungsten coil electrothermal atomic absorption spectrometry. Talanta 2007, 71, 81-89. 2 Gu, J.; Oliveira, S. R.; Donati, G. L.; Gomes Neto, J. A. and Jones, B. T. Rugged, Portable Tungsten Coil Atomic Emission Spectrometer. Analytical Chemistry 2011, 83(7), 2526-2531.

3 Liu, R.; Wu, P.; Xu, K.; Lv, Y. and Hou, X. Highly sensitive and interference-free determination of bismuth in environmental samples by electrothermal vaporization atomic fluorescence spectrometry after hydride trapping on iridium-coated tungsten coil. Spectrochimica. Acta, Part B 2008, 63B, 704-709.

4 Davis, A. C.; Alligood, B. W.; Calloway, Clifton P., J. and Jones, B. T. Direct determination of cadmium in urine by electrothermal vaporizer-inductively coupled plasma analysis using a tungsten coil vaporizer. Applied Spectroscopy 2005, 59, 13001303.

5 Donati, G. L.; Amais, R. S. and Nobrega, J. A. Tungsten coil electrothermal matrix decomposition and sample vaporization to determine P and Si in biodiesel by 
inductively coupled plasma mass spectrometry. Journal of Analytical Atomic Spectrometry 2013, 28(2), 280-287.

6 Bruhn, C. G.; Neira, J. Y.; Valenzuela, G. D. and Nobrega, J. A. Determination of cadmium in hair and blood by tungsten coil electrothermal atomic absorption spectrometry with chemical modifiers. Talanta 1999, 48, 537-549.

7 Oliveira, P. V.; Catanho, M.; Nobrega, J. A. and Luccas, P. O. Evaluation of heating programs for electrothermal atomic absorption spectrometry using a tungsten coil. Quimica Nova 2000, 23, 706-708.

8 Queiroz, Z. F.; Oliveira, P. V.; Nobrega, J. A.; Silva, C. S.; Rufini, I. A.; de Sousa, S. S. and Krug, F. J. Surface and gas phase temperatures of a tungsten coil atomizer.', Spectrochimica. Acta, Part B 2002, 57B, 1789-1799.

9 Queiroz, Z. F.; Krug, F. J.; Oliveira, P. V.; Silva, M. M. and Nobrega, J. A. Electrothermal behavior of sodium, potassium, calcium and magnesium in a tungsten coil atomizer and review of interfering effects. Spectrochimica Acta, Part B 2002, 57B, 49-61.

10 Bruhn, C. G.; Ambiado, F. E.; Cid, H. J.; Woerner, R.; Tapia, J. and Garcia, R. Analytical evaluation of a tungsten coil atomizer for cadmium, lead, chromium, manganese, nickel and cobalt determination by electrothermal atomic absorption spectrometry. Analytica Chimica Acta 1995, 306, 183-192.

11 Buckley, B. T. and Boss, C. B. A Tungsten Filament Vaporizer for Sample Introduction into a Direct-Current Plasma. Applied Spectroscopy 1990, 44(3), 505-512. 
12 Levine, K.; Wagner, K. A. and Jones, B. T. Low-cost, modular electrothermal vaporization system for inductively coupled plasma atomic emission spectrometry. Applied Spectroscopy 1998, 52, 1165-1171.

13 Donati, G. L.; Pharr, K. E.; Calloway, C. P.; Nobrega, J. A. and Jones, B. T. Determination of $\mathrm{Cd}$ in urine by cloud point extraction-tungsten coil atomic absorption spectrometry. Talanta 2008, 76, 1252-1255.

14 Bruhn, C. G.; Neira, J. Y.; Valenzuela, G. D. and Nobrega, J. A. Chemical modifiers in a tungsten coil electrothermal atomizer. Part 1. Determination of lead in hair and blood. Journal of Analytical Atomic Spectrometry 1998, 13, 29-35 15 Cresser, M. and Mullins, C. Theoretical temperature-time curves for electrically heated filament atom reservoirs : Their significance in analytical spectrometry. Analytica Chimica Acta 1974, 68(2), 377-385.

16 Navarre, E. C.; Bright, L. K.; Osterhage, A. and Lada, B. Development of an Nmethyl pyrrolidone based method of analysis for lead in paint. Analytical Methods 2012, 4(12), 4295-4302.

17 Sneddon, J. Background Correction Techniques in Atomic Spectroscopy. Spectroscopy 1987, 2(5), 38-45.

18 Desai, P. D.; Chu, T. K.; James, H. M. and Ho, C. Y. Electrical Resistivity of Selected Elements. Journal of Physical and Chemical Reference Data 1984, 13(4), 1069-1096.

19 Lide, D. R. CRC Handbook of Chemistry and Physics. 71th ed.; CRC Press: Boca Raton, FL, 1990; pp 12-107.

20 Ediger, R. D. Atomic Absorption Analysis with the Graphite Furnace using Matrix Modification. Atomic Absorption Newsletter 1975, 14, 127-130. 
21 Sturgeon, R. E. Factors Affecting Atomization and Measurement in Graphite Furnace Atomic Absorption Spectrometry. Analytical Chemistry 1977, 49, 1255A-1267A.

Table 1: Representative thermal program for the 15-V filament.

Heating Step Time (s) Current-mode (A) Voltage-mode (V)

Drying $\quad 125 \quad 2.0 \quad 2.0 \mathrm{~A}^{*}$

$\begin{array}{llll}\text { Pyrolysis } & 5 & 3.5 & 1.0\end{array}$

Pause $\quad 15 \quad 0 \quad 0$

$\begin{array}{llll}\text { Atomization } & 3 & 6.0 & 3.4\end{array}$

$\begin{array}{llll}\text { Cleaning } & 3 & 7.0 & 6.5\end{array}$

${ }^{*}$ Current control was used during drying due to the $1.0 \mathrm{~V}$ lower limit of the power supply. The $2.0 \mathrm{~A}$ current corresponds to a voltage between 0.36 and $0.40 \mathrm{~V}$.

List of captions

Figure 1 Schematic of the tungsten filament mount and glass enclosure.

Figure 2 The resistance data from several $15-\mathrm{V}$ filaments. The temperatures are equilibrium values. The data are fit to the equation $T=23.59+2716 \times R^{0.8367}$ with a value of $R^{2}=0.9999997$ 
Figure 3 The voltamperometric model compared to a blackbody radiator model in current $(A)$ and an optical pyrometry model in voltage (B). The inset shows the low voltage portion of the models.

Figure 4 Temperature traces for the 5-second pyrolysis step of a Cd sample. The power supply was set to 1.0 volt (black) and 3.5 amperes (grey). Pyrolysis begins at $123 \mathrm{~s}$ in each trace.

Figure 5 Pyrolysis and atomization curves for unmodified $12 \mu \mathrm{g} \mathrm{L}-1$ Cd samples in 0.2 $\%(\mathrm{v} / \mathrm{v}) \mathrm{HNO}_{3}$. The data in $\mathrm{A}$ are plotted against the equilibrium temperature that corresponds to the applied voltage or current during pyrolysis and atomization. The data in $\mathrm{B}$ are plotted against the temperature measured during the experiment. The integrated absorbance values from voltage-mode (squares) and current-mode (diamonds) experiments are shown with pyrolysis as closed marks and atomization open marks.

Figure 6 Pyrolysis and atomization curves for phosphate modified $12 \mu \mathrm{g} \mathrm{L}-1 \mathrm{Cd}$ samples in $0.2 \%(\mathrm{v} / \mathrm{v}) \mathrm{HNO}_{3}$. The $12-\mathrm{V}$ filament was limited to a $4 \mu \mathrm{L}$ sample size, therefore the integrated area is plotted separately on the right-hand axis. The $x$-axis is the plotted as the maximum filament temperature achieved during pyrolysis or atomization, respectively.

Figure 7 Cadmium absorbance and temperature profiles at an atomization current of $6.0 \mathrm{~A}$ (grey) and voltage of $3.4 \mathrm{~V}$ (black). The atomization step initiated at $143 \mathrm{~s}$ in both traces. The peak absorbance is marked with a vertical line. 
Figure 8 Atomization curves for phosphate modified $12 \mu \mathrm{g} \mathrm{L}-1 \mathrm{Cd}$ samples in $0.2 \%$ (v/v) $\mathrm{HNO}_{3}$. Sample volumes were $10 \mu \mathrm{L}$ for the $15-\mathrm{V}$ filament and $4 \mu \mathrm{L}$ for the $12-\mathrm{V}$ filament. 